\title{
Révolution, folklore et avant-gardes. Entretien avec Serge Fauchereau
}

\section{Paul-Henri Giraud}

\section{OpenEdition}

\section{Journals}

Édition électronique

URL : https://journals.openedition.org/ideas/1728

DOI : $10.4000 /$ ideas. 1728

ISSN : 1950-5701

Éditeur

Institut des Amériques

\section{Référence électronique}

Paul-Henri Giraud, «Révolution, folklore et avant-gardes. Entretien avec Serge Fauchereau », IdeAs [En ligne], 8 | 2016, mis en ligne le 20 décembre 2016, consulté le 18 octobre 2022. URL : http:// journals.openedition.org/ideas/1728; DOI : https://doi.org/10.4000/ideas.1728

Ce document a été généré automatiquement le 18 octobre 2022.

\section{(c) (i) $\odot$}

Creative Commons - Attribution - Pas d'Utilisation Commerciale - Pas de Modification 4.0 International - CC BY-NC-ND 4.0

https://creativecommons.org/licenses/by-nc-nd/4.0/ 


\title{
Révolution, folklore et avant- gardes. Entretien avec Serge Fauchereau
}

\author{
Paul-Henri Giraud
}

Après avoir enseigné la littérature américaine à l'Université de New York, puis du Texas, Serge Fauchereau a travaillé pendant une dizaine d'années au Centre Pompidou comme commissaire de grandes expositions. Il a poursuivi cette activité à l'étranger, notamment en Chine, Italie, Angleterre, Allemagne, Espagne et au Mexique. Parallèlement à sa vocation d'écrivain, il a été également professeur à l'Institut des hautes études en arts plastiques.

PAUL-HENRI GIRAUD : Quelle est selon vous l'originalité de cette exposition d'art mexicain à Paris?

Serge Fauchereau : La principale originalité de cette exposition est d'incorporer dans ses espaces des séquences de cinéma mexicain qui rythment et dynamisent le parcours par des temps d'arrêt au gré des visiteurs.

PHG : Le sous-titre de l'exposition met en valeur, à travers Rivera et Orozco, deux des grands muralistes mexicains. Parvient-elle selon vous à donner une idée de «l'art monumental » des muralistes en l'absence de toute peinture murale?

SF : Il me semble que l'aspect fresquiste de l'art mexicain a volontairement été laissé de côté. Une véritable fresque est indissociable de son mur (je ne suis parvenu à en amener trois à l'exposition « Mexique-Europe » de Lille en 2004 que parce que j'ai bénéficié d'un concours de circonstances et de partenaires mexicains exceptionnels). Plutôt que de frustrer le public en ne montrant que des photos ou des transpositions mobiles, on a préféré d'autres médias pour le grand public, le cinéma, par exemple, qui répond bien à l'espace du Grand Palais.

PHG : Autre élément clé du sous-titre: Frida Kahlo, dont les œuvres sont exposées aux côtés de celles d'autres «femmes fortes de la Révolution» - les photographes Tina Modotti et Lola Álvarez Bravo, les peintres Nahui Olin, María Izquierdo, Olga Costa et Rosa 
Rolanda. Cette présentation élargie des artistes femmes du Mexique de la première partie du XXe siècle vous paraît-elle convaincante?

SF : Dans un pays où la parité homme-femme est encore moins respectée que chez nous, les femmes qui résistent à cet état d'esprit sont fortes, évidemment. Elles représentaient une minorité mais c'était une bonne idée de le montrer. La présence de Rosa Rolanda est bienvenue. En revanche, j'aurais préféré un tableau de Remedios Varo plutôt que les grands bariolages de Nahui Olin. On ne pourra pas en faire une seconde Frida Kahlo: elle a été une figure pittoresque mais reste une artiste naïve peu convaincante. Frida Kahlo est aussi une figure romanesque mais d'abord une artiste authentique et singulière - même si on exagère son importance.

PHG : L'association photo / peinture / dessin / sculpture et cinéma vous semble-t-elle bien fonctionner? Permet-elle selon vous de donner à voir les avant-gardes mexicaines dans leur diversité ?

SF : D'une grande culture ou d'une grande période artistique, on voudrait tout faire connaître mais on ne peut jamais qu'en donner une idée plus ou moins juste. La musique, par exemple, est souvent la grande oubliée (ici je pense à Carrillo, à Silvestre Revueltas, à Chávez, à d'autres), tout comme les décors et costumes scéniques. Mais comment faire dans le cadre d'un musée? On comprend que le commissaire ait choisi de s'en tenir à l'art strictement plastique. La photographie pose d'autres problèmes. C'est un art très répandu, très prisé, surtout de la jeune génération. Il est indispensable de montrer les grands noms mais on ne peut être complet; ici, par exemple, où est Casasola, photographe de la révolution ? Où est Kati Horna, photographe surréaliste? C'est un phénomène général: la photographie nécessite de plus en plus des expositions spécifiques, pour elle-même.

PHG : Le surréalisme, justement, est-il bien représenté ? Comment expliquer l'absence de tout tableau de Remedios Varo ou de Gunther Gerzso?

SF : Remedios Varo et Gerzso sont assurément des absences majeures. Je songe aussi à Carlos Orozco Romero dans certaines peintures, à Amero dans ses photomontages, à Rêve et pressentiment de María Izquierdo qui, elle, est présente. Au Mexique, le surréalisme n'étant pas un mouvement fermé, organisé, il touche de nombreux artistes. Alice Rahon est très bien représentée. Carlos Mérida n'est pas oublié.

PHG : Cette exposition met-elle suffisamment en valeur ce que l'historienne de l'art Teresa del Conde a appelé «l'autre face de l'école mexicaine » - «ces artistes sans affiliation politique avec la Révolution», comme le dit Agustín Arteaga, commissaire de cette exposition (Arteaga A. (dir.), $2016: 31$ )?

SF : Je suis de l'avis de Teresa qui pensait notamment aux divers peintres compagnons des poètes Contemporáneos, Rodríguez Lozano, par exemple. L'histoire de l'art moderne procède par mouvements et par groupes constitués et néglige souvent les non-affiliés. Leur dispersion diminue leur impact.

Pour les militants révolutionnaires, on aurait pu montrer Leopoldo Méndez et quelques autres du Taller de Gráfica Popular et travailler davantage la représentation du stridentisme (Fermín Revueltas réduit à une seule petite gravure !).

PHG : L'exposition parvient-elle à éviter l'écueil mexicaniste, les clichés du "nationalisme culturel»? Parvient-elle à montrer la dimension cosmopolite de l'art mexicain? Sa dimension indigéniste sans pour autant tomber dans le folklore?

SF : On ne peut reprocher le nationalisme culturel et l'indigénisme car il me semble que c'était un des objectifs. Cela implique le survol des mouvements artistiques 
internationaux et l'insistance sur la révolution mexicaine et un folklore de musiciens, de ponchos et de sombreros. Les rituelles références à Breton et à Pollock ne cherchent pas à insérer l'art mexicain dans le mouvement international mais à montrer sa spécificité, ce qui est une intention louable.

PHG : Ne trouvez-vous pas que des artistes comme Agustín Lazo, Juan Soriano, ou même María Izquierdo et Rufino Tamayo sont insuffisamment représentés?

SF : Il vaut mieux, me semble-t-il, une seule très belle œuvre plutôt que plusieurs œuvres moins représentatives. On peut ainsi penser à Tamayo dont les œuvres ici sont toutes antérieures à 1941. À l'inverse, Soriano n'a qu'un petit tableau, curieux mais guère typique de son inspiration. María Izquierdo est une magnifique coloriste, hélas trop peu connue. Dans un monde où tout doit être rentable, l'alternative est soit de gaver un maximum de public avec ce qu'on lui a fait aimer (une exposition de Frida Kahlo tous les six mois ?) soit de lui offrir du nouveau ou ce qu'il connaît peu (Rosa Rolanda, Marius de Zayas). Le monde muséal préfère généralement une voie moyenne entre clientélisme et pédagogie.

PHG : Finalement, que souhaitez-vous retenir de cette exposition? Y a-t-il plutôt de bonnes ou de mauvaises surprises ? Des coups de cœur? Des découvertes ?...

SF : Contre les trop vastes expositions d'autrefois du type " Des Aztèques à Tamayo ", la présente exposition est moins chronologique que thématique. Elle ne néglige pas les grands: Rivera, Orozco, Siqueiros, Mérida, Tamayo... Elle veut imposer certaines images, certaines idées du Mexique et donc procède à des choix : montrer au départ l'érotisme mondain de la Belle Époque (Montenegro, Zárraga) plutôt que s'attarder sur les racines profondes (Posada avec trois documents peu mémorables), montrer la révolution dans sa réalité violente et son exaltation ultérieure plutôt que dans l'action culturelle de Best Maugard, etc. Il nous faut regarder ce qu'il y a dans l'exposition et oublier ce qu'il n'y a pas.

À titre personnel, je suis heureux de voir enfin «muséifiées » des œuvres de Cueto, d'Alva de la Canal, d'Atl que j'avais dû collecter çà et là dans des familles mexicaines pour les montrer en Europe, il y a plus de trente ans. Il y a de très bonnes surprises dans l'exposition, par exemple Le boucher de Goeritz, La passerelle de Fernández Ledesma, La coiffure de Marín, des caricatures de Covarrubias, le monument à Vénus dédié au facteur Cheval d'o'Gorman... et d'autres.

\section{BIBLIOGRAPHIE}

Arteaga, Agustín (dir.), « Mexique, 1900-1950. Diego Rivera, Frida Kahlo, José Clemente Orozco et les avant-gardes », catalogue d'exposition, Paris, RMN - Grand Palais, 2016.

Fauchereau, Serge, Bustos, le Douanier Rousseau, Posada : Des visions du Mexique, Paris, Hermann, 2016.

Fauchereau, Serge, Germán Cueto, México, RM, 2004. 
Fauchereau, Serge, Les Peintres mexicains 1910-1960, Paris, Flammarion, 2013.

Fauchereau, Serge, Los poetas surrealistas en México y Octavio Paz, México, Museo Francisco Cossío, 2008.

Fauchereau, Serge (dir.), Mexique-Europe. Allers-Retours. 1910-1960, catalogue d'exposition, Paris, Cercle d'Art, 2004.

\section{AUTEUR}

\section{PAUL-HENRI GIRAUD}

Professeur en études hispaniques contemporaines à l'Université de Lille Sciences humaines et sociales, il est directeur adjoint du Centre d'études en civilisations, langues et littératures étrangères (CECILLE) et Secrétaire général de l'Institut des Amériques. Il a publié Octavio Paz: Vers la transparence (2002, édition en espagnol revue et augmentée 2014) et Manuel Álvarez Bravo : L'Impalpable et l'imaginaire (2012). Il a récemment participé à l'édition de 1910: México entre dos épocas (2014); Le travail du visible: Claude Esteban et les arts plastiques (2014); Fotografía, cultura y sociedad en América latina. Nuevas perspectivas, numéro 219 de L'Ordinaire des Amériques (http:// orda.revues.org), décembre 2015. paul-henri.giraud@univ-lille3.fr 